\title{
Acknowledgment of Reviewers, 2010
}

The success of Phytopathology depends on the quality of manuscripts submitted by authors and on the care and competence with which they are reviewed. It is the policy of the Editorial Board to solicit reviews for manuscripts from specialists who are most qualified to review them. In addition to members of the Editorial Board, the individuals listed below provide constructive critical reviews of one or more manuscripts during the past year. Their names are published here in grateful appreciation of their contribution to the journal and to the science of phytopathology. doi:10.1094/PHYTO-101-1-0004

Abbasi, P., AAFC, London, ON, CANADA

Adaskaveg, J. E., University of California, Riverside, CA, U.S.A.

Adkins, S., U.S. Horticultural Research Laboratory, Fort Pierce, FL,

U.S.A.

Akimitsu, K., Kagawa University, Miki, Kagawa, Japan

Aldwinckle, H., Cornell University, Geneva, NY, U.S.A.

Allen, T. W., Mississippi State University, Stoneville, MS, U.S.A.

Allen, C., University of Wisconsin, Madison, WI, U.S.A.

Almeida, R., University of California, CA, U.S.A.

Alvarez, L. A., Universidad Politécnica de Valencia, Valencia,

SPAIN

Andersen, B., Technical University of Denmark, Kgs. Lyngby,

DENMARK

Anderson, J., University of Toronto, Mississauga, Ontario, CANADA

Andrivon, D., INRA, Le Rheu, FRANCE

Antoniou, P., Agricultural University Of Athens, Attica, GREECE

Appel, D., Texas A\&M University, TX, U.S.A.

Audsley, E., Cranfield University, Bedford, UNITED KINGDOM

Aziz, A., URVVC EA 2069, Reims, FRANCE

Babadoost, M., University of Illinois, Urbana, IL, U.S.A.

Bacon, C. W., USDA-ARS, Athens, GA, U.S.A.

Bai, J., Kansas State University, Manhattan, KS, U.S.A.

Bai, G., USDA, Manhattan, KS, U.S.A.

Bailey, B., Agricultural Research Service, Beltsville, MD, U.S.A.

Bajet, N., Eurofins STA Laboratories, Inc., Longmont, CO, U.S.A.

Bakker, P., Utrecht, Netherlands

Bakker, M., University of Minnesota, St. Paul, MN, U.S.A.

Balint-Kurti, P. J., USDA-ARS, Raleigh, NC, U.S.A.

Barak, J., University of Wisconsin, Madison, WI, U.S.A.

Bariana, H., University of Sydney, Narellan, New South Wales,

AUSTRALIA

Barnes, I., Forestry and Agricultural Biotechnology Institute (FABI),

Pretoria, Gauteng, SOUTH AFRICA

Barny, M.-A., INRA, Paris, FRANCE

Bayraktar, H., Ankara University, Ankara, TURKEY

Beaulieu, C., Université de Sherbrooke, Sherbrooke, Quebec, CANADA

Bennett, A., University of Warwick, UNITED KINGDOM

Bennett, R. S., USDA-ARS, Shafter, CA, U.S.A.

Bergstrom, G. C., Cornell University, Ithaca, NY, U.S.A.

Biggs, A. R., West Virginia University, Kearneysville, WV, U.S.A.

Bloemberg, G., University of Zurich, SWITZERLAND

Bock, C., USDA-ARS USHRL, Ft. Pierce, FL, U.S.A.

Boerner, A., IPK, Gatersleben, GERMANY

Bové, J. M., INRA and University Victor Segalen Bordeaux 2,

Villenave d'Ornon, FRANCE

Brien, C., University of South Australia, Adelaide, South Australia, AUSTRALIA

Brown, J. K., University of Arizona, Tucson, AZ, U.S.A.

Brown, R. L., USDA-ARS-SRRC, New Orleans, LS, U.S.A.

Brown, C. R., USDA-ARS, Prosser, WA, U.S.A.

Bull, C., USDA-ARS

Burlakoti, R. R., Nova Scotia Agricultural College, Truro, Nova

Scotia, CANADA

Burr, T., Cornell University-NYSAES, Geneva, NY, U.S.A.

Cai, G., Cornell Univeristy, Ithaca, NY, U.S.A.

Cai, K., Guangzhou, CHINA

Candela, M. C., University of Murcia, Espinardo (Murcia), Murcia,

SPAIN

Cardinale, F., University of Turin, Grugliasco, ITALY
Carisse, O., Agriculture and AgriFood CANADA, St-Jean-sur-

Richelieu, Quebec, CANADA

Carlier, J., CIRAD, Montpellier, FRANCE

Carputo, D., University of Naples, Portici, ITALY

Carris, L., Washington State University, Pullman, WA, U.S.A.

Castillo, J., PROINPA Foundation, Cochabamba, BOLIVIA

Castillo, P., Institute for Sustainable Agriculture, Cordoba, SPAIN

Ceresini, P. C., ETH Zurich-Swiss Federal Institute of Technology,

Zurich, SWITZERLAND

Chakraborty, S., CSIRO, Brisbane, Queensland, AUSTRALIA

Chastagner, G. A., Washington State University, Puyallup, WA, U.S.A.

Chen, X., USDA-ARS and Washington State University, Pullman,

WA, U.S.A.

Chikh, A., Utsunomiya University, Utsunomiya, JAPAN

Chilvers, M. I., Michigan State University, East Lansing, MI, U.S.A.

Civerolo, E. L., USDA, Parlier, CA, U.S.A.

Collinge, D. B., University of Copenhagen, Frederiksberg C, DENMARK

Cooksey, D., University of California-Riverside, CA, U.S.A.

Cooper, B., USDA-ARS, Beltsville, MD, U.S.A.

Cotty, P., USDA, U.S.A.

Cowger, C., USDA-ARS, Raleigh, NC, U.S.A.

Cox, K. D., Cornell University, Geneva, NY, U.S.A.

Crouch, J. A., Rutgers University, New Brunswick, NJ, U.S.A. Cubeta, M., North carolina State University, Raleigh, NC, U.S.A.

Daayf, F., University of Manitoba, Winnipeg, Manitoba, CANADA

Davis, K., Rothamsted Research, Harpenden, UNITED KINGDOM

Dawson, W. O., University of Florida, Lake Alfred, FL, U.S.A.

De Boer, S., CFIA, Charlottetown, Prince Edward Island, CANADA de la Fuente, L., Auburn University, Auburn, AL, U.S.A.

de Sa, P., USDA/APHIS, Beltsville, MD, U.S.A.

De Wolf, E., KSU, Manhattan, KS, U.S.A.

del Rio, L., North Dakota State University, Fargo, ND, U.S.A.

Delmotte, F., INRA, Villenave d'Ornon Cedex, FRANCE

Deom, M., University of Georgia, Athens, Georgia, U.S.A.

Dermastia, M., National Institut of Biology, Ljubljana, SLOVENIA

Dhillon, B., University of British Columbia, Vancouver, British

Columbia, CANADA

Di Serio, F., Consiglio Nazionale delle Ricerche, Bari, ITALY

Díaz Varela, J., University of A Coruna, A Coruna, SPAIN

Dijksterhuis, J., CBS, Utrecht, NETHERLANDS

Ding, B., Ohio State University, Columbus, OH, U.S.A.

Domier, L., USDA-ARS, Urbana, IL, U.S.A.

Donofrio, N., University of Delaware, Newark, U.S.A.

Dorrance, A. E., The Ohio State University, Wooster, OH, U.S.A.

Douches, D., East Lansing, MI, U.S.A.

Douhan, G. W., University of California, Riverside, CA, U.S.A.

Downer, A. J., University of California, Ventura, CA, U.S.A.

du Toit, L. J., Washington State University, Mount Vernon, WA, U.S.A.

Duffy, S., Rutgers, The State University of New Jersey, New Brunswick, NJ, U.S.A.

Dung, J., Washington State University, Pullman, WA, U.S.A.

Eastwell, K., Prosser, U.S.A.

Edwards, M., USDA-ARS, Fargo, ND, U.S.A.

Eibach, R., JKI-Institute for Grapevine Breeding Geilweilerhof,

Siebeldingen, GERMANY

Elad, Y., Volcani Center, Bet Dagan, ISRAEL 
Elphinstone, J., Department for Food, Environment and Rural Affairs, York, UNITED KINGDOM

English, J., University of Missouri, Columbia, MO, U.S.A.

Epstein, L., Davis, CA, U.S.A.

Esker, P., University of Wisconsin, Madison, WI, U.S.A.

Evans, K., University of Tasmania, New Town, Tasmania,

AUSTRALIA

Eveillard, S., INRA, Villenave d'Ornon cedex, FRANCE

Faize, M., National Institute for Agro-Environmental Sciences,

Tsukuba, Ibaraki, JAPAN

Fakhoury, A. M., Southern Illinois University, Carbondale, IL, U.S.A.

Falk, B., University of California, Davis, CA, U.S.A.

Fargette, D.

Farman, M., University of Kentucky, Lexington, KY, U.S.A.

Fauquet, C., Danforth Plant Science Center, St. Louis, MO, U.S.A.

Feau, N., INRA, Cestas Cedex, FRANCE

Ferrandino, F. J., The Connecticut Agricultural Experiment Station,

New Haven, CT, U.S.A.

Flaherty, J., Coker College, Hartsville, SC, U.S.A.

Fletcher, J., Oklahoma State University, Stillwater, OK, U.S.A.

Foissac, X., INRA, Université de Bordeaux2, Villenave d'Ornon,

FRANCE

Forbes, G., Lima, PERU

Fraaije, B., Rothamsted Research, Harpenden, Hertfordshire,

UNITED KINGDOM

Fravel, D., USDA-ARS, Beltsville, MD, U.S.A.

Frederick, R., USDA-ARS

Freeman, S., Bet Dagan, ISRAEL

Fry, W., Cornell University, Ithaca, NY, U.S.A.

Fuchs, M., Cornell University, Geneva, NY, U.S.A

Fukui, R., Utsunomiya University, Utsunomiya, Tochigi, JAPAN

Fulbright, D. W., Michigan State University, East Lansing, MI,

U.S.A.

Gadoury, D., Cornell University, NYSAES, Geneva, NY, U.S.A.

Garbelotto, M., University of California, Berkeley, CA, U.S.A.

Garcia-Arenal, F., Universidad Politecnica de Madrid, Madrid,

Madrid, SPAIN

Geiser, D., Penn. State University, University Park, PA, U.S.A.

Gent, D. H., USDA-ARS, Corvallis, CO, U.S.A.

Georgi, L., Rutgers University, Chatsworth, NJ, U.S.A.

Ghabrial, S., University of Kentucky, Lexington, KY, U.S.A.

Gheysen, G., Ghent, Belgium, GERMANY

Gilbert, J., Cereal Research Centre, Winnipeg, Manitoba, CANADA

Gilbertson, R., University of California, Davis, CA, U.S.A.

Gisi, U., Syngenta Crop Protection, Stein, SWITZERLAND

Gladders, P., Adas Boxworth, Cambridge, UNITED KINGDOM

Glenn, A. E., USDA-ARS, Athens, GA, U.S.A.

Goates, B., USDA-ARS, Aberdeen, ID, U.S.A.

Goheen, E., USDA FS, Central Point, U.S.A.

Gold, S. E., University of Georgia, Athens, GA, U.S.A.

Gomes, E., Universidade Estadual paulista, São José do Rio Preto,

São Paulo, BRAZIL

Goodwin, S., USDA-ARS, West Lafayette, Indiana, U.S.A.

Goswami, R., North Dakota State University, Fargo, ND, U.S.A.

Graham, J. H., Citrus Research and Education Center, Lake Alfred,

FL, U.S.A.

Gray, S., USDA-ARS, Cornell University, Ithaca, NY, U.S.A.

Grenier, E., INRA, Le Rheu, FRANCE

Grove, G. G., Washington State University, Prosser, WA, U.S.A.

Gubler, W., UC Davis, Davis, California, U.S.A.

Gudmestad, N. C., North Dakota State University, Fargo, ND, U.S.A.

Guidot, A., INRA, Castanet Tolosan, FRANCE

Guttman, D. S., University of Toronto, Toronto, Ontario, CANADA

Hammond, R., USDA-ARS, Beltsville, MD, U.S.A.

Hansen, E., Oregon State University, Corvallis, OR, U.S.A.

Hanson, L., USDA/ARS, East Lansing, MI, U.S.A.

Hardham, A., The Australian National University

Harper, S., MAF Biosecurity NZ, Auckland, NEW ZEALAND

Harrington, T. C., Iowa State University, Ames, IA, U.S.A.

Harrison, N., University of Florida, Fort Lauderdale, FL, U.S.A.
Hartman, G., USDA-ARS and University of Illinois, Urbana, IL, U.S.A.

Haynes, K., USDA, Beltsville, MD, U.S.A.

Hietala, A. M., Norwegian Forest and Landscape Insitute, Aas, NORWAY

Hillman, Brad, Rutgers University, New Brunswick, NJ, U.S.A.

Höfte, M., Ghent University, Gent, Belgium, GERMANY

Hokanson, S., University of Minnesota, St. Paul, MN, U.S.A.

Holb, I. J., University of Debrecen, Debrecen, HUNGARY

Hopkins, D., University of Florida, Apopka, FL, U.S.A.

Hou, A., Agriculture and Agri-Food CANADA, Morden, Manitoba, CANADA

Hu, X., SAIC-Frederick, Frederick, MD, U.S.A.

Huang, Y.-J., Rothamsted Research, Harpenden, Hertfordshire, UNITED KINGDOM

Huettel, R., Auburn University, AL, U.S.A.

Hughes, T., University of Wisconsin, Madison, WI, U.S.A.

Hunger, R., Oklahoma State University, Stillwater, OK, U.S.A.

Ishimaru, C., University of Minnesota, St. Paul, MN, U.S.A.

Ivors, K., NC State University, Fletcher, NC, U.S.A.

Iwanami, T., National Institute of Fruit Tree Science, Tsukuba,

Ibaraki, JAPAN

Jacobs, J., Michigan State University, E. Lansing, MI, U.S.A.

Jacquot, E., INRA, Le Rheu, FRANCE

Jansen, V., Royal Holloway University of London

Jarausch, W., AlPlanta RLP Agroscience GmbH, Neustadt/W., GERMANY

Jarosz, A. M., Michigan State University, East Lansing, MI, U.S.A.

Jaspers, M., Lincoln University, Lincoln, NEW ZEALAND

Jena, K. K., International Rice Research Institute, Makati City, Kyeonggi, REPUBLIC OF KOREA

Jenkins, D. M., University of Hawaii, Honolulu, HI, U.S.A.

Jiménez-Díaz, R. M., Instituto Agricultura Sostenible, Córdoba, Córdoba, SPAIN

Jimenez-Gasco, M., The Pennsylvania State University, University Park, PA, U.S.A.

Johnson, Ken, Dept. Botany and Plant Pathology, Corvallis, OR, U.S.A.

Johnson, D. A., Washington State University, Pullman, WA, U.S.A.

Jones, R., Agricultural Ressearch Western AUSTRALIA, Perth,

Western Australia, AUSTRALIA

Jones, J., Scottish Crop Research Institute, Dundee, UNITED KINGDOM

Judelson, H., University of California, Riverside, CA, U.S.A.

Kachroo, P., University of Kentucky, Lexington, KY, U.S.A.

Kassemeyer, H.-H., Staatliches Weinbauinstitut, Freiburg im Breisgau, GERMANY

Katan, J., Hebrew University, Rehovot, ISRAEL

Keeping, M., South African Sugarcane Research Institute, Mount Edgecombe, KZN, SOUTH AFRICA

Keith, L., USDA-ARS, Hilo, HI, U.S.A.

Keller, B., University of Zurich, Zurich, SWITZERLAND

Kelly, J., Michigan State University, East Lansing, MI, U.S.A.

Kenyon, D., Edinburgh, UNITED KINGDOM

Kerlan, C., University of Idaho, Moscow, ID, U.S.A.

Kikuchi, T., Forestry and Forest Product Research Institute, Tsukuba, Ibaraki, JAPAN

Kim, D.-H., Institute for Molecular Biology and Genetics, Basic

Science Research Insititute, KOREA

Kinkel, L. L., University of Minnesota, St. Paul, MN, U.S.A.

Kisha, T., USDA-ARS, Pullman, WA, U.S.A.

Kiss, L., Plant Protection Institute of the Hungarian Academy of

Sciences, Budapest, HUNGARY

Klosterman, S. J., USDA-ARS, Salinas, CA, U.S.A.

Kobayashi, D. Y., Rutgers University, New Brunswick, NJ, U.S.A.

Koenig, R., Julius Kühn Institute, Braunschwweig, GERMANY

Kohn, L., University of Toronto, Mississauga, Ontario, CANADA

Koike, S., University of California, Salinas, CA, U.S.A.

Kolmer, J., USDA-ARS, St. Paul, MN, U.S.A.

Kousik, S., U.S. Vegetable Laboratory, Charleston, SC, U.S.A.

Kox, L. F. F., Plant Protection Service, Wageningen,

NETHERLANDS 
Kreuze, J., International Potato Center, Lima, PERU

Kreuze, H., International Potato Center (CIP), Lima, PERU

Kuldau, G., Penn. State, University Park, PA, U.S.A.

Lamour, K., University of Tennessee, Knoxville, TN, U.S.A.

Lannou, C., INRA, Thiverval Grignon, FRANCE

Lawrence, C., Blacksburg, Virginia, U.S.A.

Lazarovits, G., Agric. \& Agri-Food CANADA, London, Ontario,

CANADA

Lebecka, R., Plant Breeding and Acclimatization Institute, Mlochow, POLAND

Lees, A., SCRI, Dundee, UNITED KINGDOM

Legorburu, J., NEIKER, VITORIA/GASTEIZ, Basque Country, SPAIN

Leslie, J. F., Kansas State University, Manhattan, KS, U.S.A.

Levy, M., Faculty of agriculture, Rehovot, ISRAEL

Lewers, K., USDA-ARS-BARC, Beltsville, MD, U.S.A.

L'homme, J.-P., IRD, Montpellier, FRANCE

Li, W., USDA APHIS, Beltsville, MD, U.S.A.

Liang, Y., Chinese Academy of Agricultural Sciences, Beijing, CHINA

Linde, C., AUSTRALIAn National University, Canberra,

AUSTRALIA

Lindow, S., University of California, Berkeley, CA, U.S.A.

Ling, K.-S., USDA-ARS, Charleston, SC, U.S.A.

Liu, H.-Y., USDA-ARS, Salinas, CA, U.S.A.

Liu, Z., North Dakota State University, Fargo, ND, U.S.A.

Llop, P. F., IVIA, Moncada, Valencia, SPAIN

Loper, J., USDA-ARS, Corvallis, OR, U.S.A.

Lorenzen, J., IITA, Kampala, UGANDA

Loria, R., Cornell University, Ithaca, NY, U.S.A.

Lozovaya, V. V., University of Illinois, Urbana, IL, U.S.A.

Lu, S., Mississippi State University, Mississippi State, U.S.A.

Lukens, L., University of Guelph, Guelph, Ontario, CANADA

Lulai, E., USDA-ARS, Northern Crop Science Lab., Fargo, ND, U.S.A.

Luster, D., USDA-ARS, Ft. Detrick, MD, U.S.A.

Lutz, M., Zurich University of Applied Sciences, Wädenswil, SWITZERLAND

Lyngkjaer, M., Copenhagen University, Faculty of Life Sciences, Frederiksberg, DENMARK

Ma, Z., Hangzhou, CHINA

Madden, L. V., Ohio State University, Wooster, OH, U.S.A.

Magill, C., Texas A\&M, College Station, TX, U.S.A.

Mahaffee, W., USDA-ARS HCRL, Corvallis, OR, U.S.A.

Maloney, P., University of California, Davis, CA, U.S.A.

Marçais, B., INRA, Champenoux, FRANCE

Marek, S., Oklahoma State University, Stillwater, OK, U.S.A.

Marion, G., Biomathematics \& Statistics Scotland, Edinburgh, UNITED KINGDOM

Marois, J. J., University of Florida, Quicny, FL, U.S.A.

Marra, R. E., Connecticut Agricultural Experiment Station, New

Haven, CT, U.S.A.

Martin, F. N., USDA-ARS, Salinas, CA, U.S.A.

Matheron, M. E., University of Arizona, Yuma, AZ, U.S.A.

Maurhofer, M., Swiss Federal Institute of Technology Zürich, SWITZERLAND

Mazzola, M., USDA-ARS, Wenatchee, WA, U.S.A.

Mbofung, G., Iowa State University, Ames, IA, U.S.A.

McCartney, C., University of Saskatchewan, Saskatoon,

Saskatchewan, CANADA

McDonald, M. R., University of Guelph, Guelph, Ontario, CANADA

McDonald, B. A., Institute of Integrative Biology, ETH Zurich,

Zurich, SWITZERLAND

McGrath, M., Michigan State University, East Lansing, MI, U.S.A.

McManus, P. S., University of Wisconsin, Madison, WI, U.S.A.

McMullen, M., North Dakota State University, Fargo, ND, U.S.A.

McPhee, K., North Dakota State University, Fargo, ND, U.S.A.

McSpadden Gardener, B. B., Ohio State University-OARDC,

Wooster, OH, U.S.A.

Mehl, H. L., USDA-ARS, University of Arizona, Tucson, AZ, U.S.A.

Melcher, U., Oklahoma State University, Stillwater, OK, U.S.A.
Meng, B., University of Guelph, Guelph, Ontario, CANADA

Menzies, J., Agriculture and Agri-Food CANADA, Winnipeg,

Manitoba, CANADA

Merckling, T., Agraquest, Lyon, FRANCE

Michailides, T., University of California, Kearney Agricultural

Center, Parlier, CA, U.S.A.

Michel, V., Agroscope ACW, Conthey, SWITZERLAND

Miedaner, T., Universitaet Hohenheim, Stuttgart, GERMANY

Mila, A., North Carolina State University, Raleigh, NC, U.S.A.

Miles, M., Champaign, IL, U.S.A.

Milgroom, M. G., Cornell University, Ithaca, NY, U.S.A.

Miller, A. H., University of Kansas, Lawrence, KS, U.S.A.

Mitchell, T., The Ohio State University, Columbus, OH, U.S.A.

Mizubuti, E., Universidade Federal de Vicosa, Vicosa, MG, BRAZIL

Montesinos, E., University of Girona, Girona, GIRONA, SPAIN

Morin, L., CSIRO Entomology, Canberra, AUSTRALIA

Mundt, C. C., Oregon State University, Corvallis, OR, U.S.A.

Mundt, C., Oregon State University, Corvallis, OR, U.S.A.

Munkvold, G., Iowa State University, Ames, IA, U.S.A.

Murphy, J. F., Auburn University, AL, U.S.A.

Musetti, R., Università di Udine, Udine, Udine, ITALY

Nakajima, T., National Agricultural Research Center for Kyushu-

Okinawa Region, Koshi, Kumamoto, JAPAN

Navas-Castillo, J., CSIC, Algarrobo-Costa, Málaga, SPAIN

Nelson, E. B., Cornell University, Ithaca, NY, U.S.A.

Nelson, R., Samuel Roberts Noble Foundation, Inc., Ardmore, OK, U.S.A.

Ngugi, H. K., Penn State University, Biglerville, PA, U.S.A

Nicholson, P., John Innes Research Centre, Norwich, UNITED KINGDOM

Nischwitz, C., The University of Arizona, Tucson, AZ, U.S.A.

Nissinen, R., University of Groningen, Haren, NETHERLANDS

Nita, M., Virginia Tech, Winchester, VA, U.S.A.

Norelli, J. L., USDA-ARS, Kearneysville, WV, U.S.A.

Notteghem, J.-L., Agro. M, Montpellier Cedex 5, FRANCE

Nutter, F., Weston, Iowa State University, Ames, IA, U.S.A.

Oancea, F., Research Institute for Plant Protection, Bucharest, ROMANIA

Obradovic, A., Faculty of Agriculture, Belgrade-Zemun, SERBIA

Ojiambo, P. S., North Carolina State University, Raleigh, NC, U.S.A.

Olatinwo, R., University of Georgia, Griffin, GA, U.S.A.

Oliva, R., The Sainsbury Laboratory, Norwich, UNITED KINGDOM

Olivain, C., INRA, Dijon, FRANCE

Oliver, R. P., Murdoch University, Perth, Western AUSTRALIA, AUSTRALIA

Oudemans, P. V., Rutgers University, Chatsworth, NJ, U.S.A.

Owens, R., Beltsville Agricultural Research Center, Beltsville, MD, U.S.A.

Palumbo, J. D., USDA, ARS, Albany, CA, U.S.A.

Panthee, D., North Carolina State University, Mills River, NC, U.S.A.

Pappu, H., Washington State University, Pullman, WA, U.S.A.

Parke, J., Oregon State, Corvallis, OR, U.S.A.

Pataky, J., University of Illinois, Urbana, IL, U.S.A.

Paul, P. A., Ohio State University, Wooster, OH, U.S.A.

Paulitz, T. C., USDA-ARS, Pullman, WA, U.S.A.

Payne, G., North Carolina State, NC, U.S.A.

Peever, T., Washington State University, Pullman, WA, U.S.A.

Perazzolli, M., IASMA Research and Innovation Center, Fondazione Edmund Mach, Michele all'Adige, Trento, ITALY

Pereira, F. V., EMBRAPA Agricultural Instrumentation, São Carlos, São Paulo, BRAZIL

Peres, N., University of Florida, Wimauma, FL, U.S.A.

Pérez-Vich, B., Institute for Sustainable Agriculture (CSIC),

Cordoba, SPAIN

Peros, J.-P., INRA- Montpelier, Montpellier, FRANCE

Pertot, I., FEM, S. Michele all'Adige, TN, ITALY

Petit, E., INRA, Le Rheu, FRANCE

Pfender, W., Corvallis, OR, U.S.A.

Phillips, M., SCRI, Dundee, Fife, UNITED KINGDOM

Pickup, J., SASA, Edinburgh, UNITED KINGDOM 
Pierson, L. S., University of Arizona, Tucson, Arizona, U.S.A. Plummer, K., La Trobe University

Polston, J. E., University of Florida, Gainesville, FL, U.S.A.

Ponz, F., INIA, Pozuelo de Alarcón, Madrid, SPAIN

Pothier, J. F., Swiss Federal Research Station, Wädenswil, SWITZERLAND

Proctor, R., USDA-ARS NCAUR, Peoria, IL, U.S.A.

Prospero, S., WSL, Birmensdorf, SWITZERLAND

Prusky, D., Volcani Center, Rishon Le Tzion, ISRAEL

Pryor, B., Tucson, AZ, U.S.A.

Pryor, B. M., University of Arizona, Tucson, AZ, U.S.A.

Punja, Z. K., Simon Fraser University, Burnaby, British Columbia,

CANADA

Pusey, L., USDA-ARS, Wenatchee, Washington, U.S.A.

Queiroz, M., Universidade Federal de Viçosa, Viçosa, Minas Gerais,

BRAZIL

Randhawa, H., AAFC, Lethbridge, Alberta, CANADA

Rayapati, N., Washington State University, Prosser, WA, U.S.A.

Redinbaugh, M., USDA-ARS Corn and Soybean Research, Wooster, OH, U.S.A.

Reid, A., SASA, Edinburgh, UNITED KINGDOM

Rémus-Borel, Wilfried, Laval University, Quebec, Quebec,

CANADA

Restrepo, S., Universidad de los Andes, Bogotá, COLOMBIA

Reverberi, M., Università Sapienza, Roma, ITALY

Rezzonico, F., ACW Waedenswil, Waedenswil, ZH,

SWITZERLAND

Rioux, D., NRCan, Québec, Quebec, CANADA

Rizzo, D. M., University of California, Davis, CA, U.S.A.

Roberts, P., University of Florida, Immokalee, Florida, U.S.A.

Rosenberger, D., Cornell University/NYAES, Highland, NY, U.S.A.

Rotenberg, D., Kansas State University, Manhattan, KS, U.S.A.

Rouse, M. N., USDA-ARS Cereal Disease Laboratory, St. Paul, MN, U.S.A.

Rowhani, A., UC Davis, Davis, CA, U.S.A.

Rudd, J., Rothamsted Research, Harpenden, UNITED KINGDOM

Rupe, J. C., University of Arkansas, Fayetteville, AK, U.S.A.

Rush, C. M., TAES, Bushland, TX, U.S.A.

Rybicki, E., University Cape Town, Cape Town, WC, SOUTH AFRICA

Sanfacon, H., Agriculture and Agri-Food CANADA, Summerland,

British Columbia, CANADA

Savary, S., IRRI, Los Banos, Laguna, PHILIPPINES

Schaafsma, A., University of Guelph, Ridgetown, Ontario, CANADA

Scherm, H., University of Georgia, Athens, GA, U.S.A.

Schnabel, G., Clemson University, Clemson, SC, U.S.A.

Schneider, B., Julius Kuehn Institute, Dossenheim, GERMAY

Schneider, S., Agroscope Reckenholz-Tänikon Research Station

ART, Zurich, SWITZERLAND

Schneider, R. W., Louisiana State University, Baton Rouge, LA,

U.S.A.

Schneider, W., USDA, Fort Detrick, MD, U.S.A.

Schroeder, K., USDA-ARS, Pullman, WA, U.S.A.

Schubert, T., FL Dept. of Ag \& Consumer Serv, Gainesville, FL, U.S.A.

Scortichini, M., CRA-Istituto Sperimentale per la Frutticoltura,

Roma, ITALY

Scott, B., Massey University, Palmerston North, NEW ZEALAND

Seem, R. C., Cornell University, NYSAES, Geneva, NY, U.S.A.

Seemueller, E., Julius Kuehn Institute (JKI), Federal Research Centre

for Cultivated Plants, Dossenheim, GERMANY

Sether, D. M., University of Hawaii, Honolulu, HI, U.S.A.

Shamoun, S. F., Canadian Forest Service, Victoria, British Columbia,

CANADA

Shatters, R., USDA-ARS, USHRL, Fort Pierce, FL, U.S.A.

Shaw, M., University of Reading, Reading, UNITED KINGDOM

Sherwood, J., University of GA, Athens, GA, U.S.A.

Shipp, L., Agriculture and Agri-Food CANADA, Harrow, Ontario,

CANADA

Shtienberg, D., ARO, the Vocani Center, Bet Dagan, ISRAEL

Singh, P. K., North Dakota State University, Fargo, ND, U.S.A.
Sipes, B., University of Hawaii, Honolulu, HI, U.S.A.

Sisterson, M., USDA-ARS, Parlier, CA, U.S.A.

Slininger, P., NCAUR, Peoria, IL, U.S.A.

Slippers, B., FABI, University of Pretoria, Pretoria, SOUTH AFRICA

Smart, C. D., Cornell University, Geneva, NY, U.S.A.

Smits, T. H. M., ACW, Wädenswil, SWITZERLAND

Sosnowski, M., SARDI, Adelaide, South Australia, AUSTRALIA

Stefani, F., Université Laval, Québec, Quebec, CANADA

Stein, J. M., South Dakota State University, Brookings, SD, U.S.A.

Stenger, D., C, USDA-ARS, Parlier, CA, U.S.A.

Stevens, M., Broom'Barn Research Station, Bury St. Edmunds,

Suffolk, UNITED KINGDOM

Stone, J. K., Oregon State University, Corvallis, OR, U.S.A.

Strelkov, S., University of Alberta, Edmonton, Alberta, CANADA

Sudarshana, M., UC Davis, Davis, CA, U.S.A.

Suh, S.-O., American Type Culture Collection (ATCC), Manassas, VA, U.S.A.

Sundin, G. W., Michigan State University, East Lansing, MI, U.S.A.

Sunter, G., University of Texas at San Antonio, San Antonio, TX, U.S.A.

Sutton, T. B., North Carolina State Universtiy, Raleigh, NC, U.S.A.

Suzuki, N., Okayama University, Kurashiki, JAPAN

Svircev, A. M., Agriculture and Agri-Food CANADA, Vineland

Station, Ontario, CANADA

Szabo, L. J., USDA-ARS, St. Paul, MN, U.S.A.

Tatineni, S., USDA-ARS, Lincoln, NE, U.S.A.

Taylor, P., The University of Melbourne, Melbourne, Victoria, AUSTRALIA

Tekauz, A., Agriculture and Agri-Food CANADA (AAFC) in Winnipeg, Winnipeg, Manitoba, CANADA

Timmer, P., Citrus Research and Education Center, Lake Alfred, FL, U.S.A.

Tolin, S. A., Virginia Tech, Blacksburg, VA, U.S.A.

Tomlinson, J., Central Science Laboratory, York, UNITED KINGDOM

Torres, M. A., Universidad Politecnica de Madrid, Pozuelo de Alarcon, SPAIN

Travadon, R., University of California, Davis, CA, U.S.A.

Turechek, W. W., USDA ARS USHRL, Fort Pierce, FL, U.S.A.

Turina, M., CNR, Torino, ITALY

Tzanetakis, I., University of Arkansas, Fayetteville, AK, U.S.A.

Tzortzakakis, E. A., NAGREF, Heraklion, Crete, GREECE

Upchurch, G., USDA-ARS, Raleigh, NC, U.S.A.

Vaillancourt, L. J., University of Kentucky, Lexington, KY, U.S.A.

Valent, B., Kansas State University, Manhattan, KS, U.S.A.

Valkonen, J., University of Helsinki, Helsinki, FINLAND

Vallad, G. E., University of Florida, Wimauma, FL, U.S.A.

van den Bosch, F., Rothamsted Research, Harpenden, UNITED

KINGDOM

Van der Vlugt, R., Plant Research International, Wageningen, NETHERLANDS

van Iersel, M., University of Georgia, Athens, GA, U.S.A.

van Overbeek, L., Wageningen UR, Wageningen, NETHERLANDS

Varsani, A., University of Canterbury, Christchurch, South Africa

Venturi, V., ICGEB, Trieste, ITALY

Vera Cruz, C., International Rice Research Institute, Metro Manila, PHILIPPINES

Vidaver, A., University of Nebraska, Lincoln, NE, U.S.A.

Vincelli, P., University of Kentucky, Lexington, KY, U.S.A.

Vogel, J., Western Regional Research Center, Albany, CA, U.S.A.

Vogler, D. R., USDA, FS, PSW Research Station, Placerville, CA, U.S.A.

Voleti, S. R., Directorate of Rice Research, Hyderabad, INDIA

Walters, D. R., Scottish Agricultural College, Edinburgh, UNITED KINGDOM

Wang, Y., Nanjing Agricultural University, Nanjing, Jiangsu, CHINA

Wang, G., Ohio State University, Columbus, OH, U.S.A.

Wang, N., University of Florida, Lake Alfred, FL, U.S.A.

Wang, D., Michigan State University, East Lansing, MI, U.S.A.

Wanner, L. A, USDA-Agricultural Research Service, Beltsville, MD, U.S.A. 
Ward, T., USDA-ARS, Peoria, IL, U.S.A.

Waters, C., East Lansing, MI, U.S.A.

Wayadande, A. C., Oklahoma State University, Stillwater, OK,

U.S.A.

Wei, Y. D., University of Saskatchewan, Saskatoon, Saskatchewan, CANADA

Weller, D. M., Washington State University, Pullman, WA, U.S.A. Widmer, F., Research Station ART, Zurich, SWITZERLAND

Will, T., Justus-Liebig-University Giessen, Giessen, GERMANY

Willocquet, L., International Rice Research Institute (IRRI), Metro Manila, PHILIPPINES

Wingfield, B., University of Pretoria, Pretoria, SOUTH AFRICA

Woo, S., University of Naples, Portici (NA), ITALY
Workneh, F., Texas Agricultural Experiment Station, Bushland, TX, U.S.A.

Xiao, C.-L., Washington State University, Wenatchee, WA, U.S.A. Xiong, Z., University of Arizona, Tucson, AZ, U.S.A.

$\mathrm{Xu}, \mathrm{M}$., China Agricultural University, Beijing, CHINA

$\mathrm{Xu}, \mathrm{X}$., East Malling Research, West Malling, Kent, UNITED KINGDOM

Yamaoka, Y., Life and Environmental Sciences, Tsukuba, Ibaraki, JAPAN

Yan, L., OSU, Stillwater, OK, U.S.A.

Yuen, J. E., Swedish University of Agric. Science, Uppsala, SWEDEN

Zhang, H., Nanjing Agricultural University, Nanjing, CHINA

Zhao, Y., University of Illinois, Urbana, IL, U.S.A. 